\title{
Erratum to: Comparative transcriptome analyses on silk glands of six silkmoths imply the genetic basis of silk structure and coloration
}

\author{
Yang Dong ${ }^{1,2+}$, Fangying Dai ${ }^{3 \dagger}$, Yandong Ren ${ }^{2 \dagger}$, Hui Liu ${ }^{2 \dagger}$, Lei Chen ${ }^{2 \dagger}$, Pengcheng Yang ${ }^{4}$, Yanqun Liu ${ }^{5}$, Xin Li ${ }^{6}$, \\ Wen Wang ${ }^{2^{*}}$ and Hui Xiang ${ }^{2^{*}}$
}

\section{Erratum}

After the publication of this work [1], we were requested to make a supplementary note clarifying the sampling of the silkmoths used in our study, especially Antheraea assama. Larvae of A. pernyi, Samia Cynthia ricini and A. yamamai were provided by Shenyang Agricultural University, Hunan Nanlingence and technology development CO.LTD (https://hnnltckj.cn.china.cn), respectively. Larvae of Actias selene and Rhodinia newara was provided by Dr. Yun Wu, a specialist on ecology of butterflies and silk moths [2]. Antheraea assama, was collected in Xishuangbanna, Yunnan Province, China, and reared indoor with Dr. Yun Wu's kind help. In his book [2], Dr. $\mathrm{Wu}$ also illustrated A. assama in Yunnan province, China. As to collection and rearing of this species, briefly, moths in the wild are collected by a light trap [2]. Pregnant female moths were then brought indoor and kept at room temperature. Eggs were laid in a container and collected and disinfected. The eggs were then hatched in incubator at $28{ }^{\circ} \mathrm{C}$. The larvae were fed with fresh leaves with branches of a host plant Cinnamomum japonicum until the 5th instar, when it is ready for sampling. Antheraea assama is an important economic insect in India. These species also inhabits Yunnan province, China [2, 3]. Recently, Chinese researchers have been making efforts on artificial rearing this species and now it can be reared in laboratory condition [2, 4-6] (Additional file 1: Figure S1).

We regret any inconvenience that this inaccuracy might have caused.

\footnotetext{
* Correspondence: wwang@mail.kiz.ac.cn; xiangh@mail.kiz.ac.cn

${ }^{\dagger}$ Equal contributors

${ }^{2}$ State Key Laboratory of Genetic Resources and Evolution, Kunming Institute of Zoology, Chinese Academy of Sciences, 32 East Jiaochang Road, Kunming, Yunnan Province 650223, China
}

\section{Additional file}

Additional file 1: Figure S1. Antheraea assama in Yunnan province, China. A. Eggs and newly hatched larvae. B. The fifth instar larva. C. Male (left) and female (right) Pupae. D. Mating Adults. Cocoons (E) and female moths ready for laying eggs $(F)$ are harvested by indoor rearing. All the photos were taken by Dr. Zhong J. Copyrights are asserted and protected ( $213 \mathrm{~kb}$ )

\begin{abstract}
Author details
${ }^{1}$ Kunming University of Science and Technology, 727 South Jingming Road, Chenggong District, Kunming, Yunnan Province 650500, China. ${ }^{2}$ State Key Laboratory of Genetic Resources and Evolution, Kunming Institute of Zoology, Chinese Academy of Sciences, 32 East Jiaochang Road, Kunming, Yunnan Province 650223, China. ${ }^{3}$ State Key Laboratory of Silkworm Genome Biology, Key Sericultural Laboratory of Agricultural Ministry, Institute of Sericulture and Systems Biology, Southwest University, 2 Tiansheng Road, Beibei District, Chongqing 400715, China. ${ }^{4}$ Institute of Zoology, Chinese Academy of Sciences, 69 East Beichen Road, Chaoyang District, Beijing 100101, China. ${ }^{5}$ Shenyang Agricultural University, 120 Dongling Road, Shenhe District, Shenyang, Shenyang Province 110866, China. ${ }^{6}$ Center for Epigenetics, Johns Hopkins University School of Medicine, Baltimore, MD 21205, USA.
\end{abstract}

Received: 13 July 2017 Accepted: 13 July 2017

Published online: 21 July 2017

\section{References}

1. Dong Y, Dai FY, Ren YD, Liu H, Chen L, Yang PC, Liu YQ, Li X, Wang W, Xiang $\mathrm{H}$. Comparative transcriptome analyses on silk glands of six silkmoths imply the genetic basis of silk structure and coloration. BMC Genomics. 2015;16:203. doi:10.1186/s12864-015-1420-9.

2. Wu Y. The marvelous moths of China. Zhengzhou: Henan Science and Technology Press; 2017.

3. Editorial Committee of Fauna Sinica. Academia Sinica. Fauna Sinica, vol. 5, Lepidoptera Bombycidae, Saturniidae, Thyrididae. Beijing: Science Press; 1996.

4. Zhong J, Jiang XJ, Chen A, Tang F, Shao Y, Chai J, Bai X. Gene cloning and phylogenetic analysis of mitochondrial DN a COI of Antheraea Assama collected in Yunnan province. Sci Sericulture. 2014;40(4):0627-32.

5. Z Zhong J, Dong ZP, Jiang XJ, LI T. Bionomics of Antheraea Assamensis. Chin J Appl Entomol. 2013;50(3):800-6.

6. Shri M. Muga in China! India Silk. 2015;2015(5):28-9. 